\title{
Quasi-phase-matched four-wave mixing of optical pulses in periodically modulated silicon photonic wires
}

\author{
Spyros Lavdas ${ }^{a}$, Jeffrey B. Driscoll ${ }^{b}$, Richard R. Grote ${ }^{b}$, Richard M. Osgood, Jr. ${ }^{b}$, and \\ Nicolae C. Panoiu ${ }^{a}$ \\ ${ }^{a}$ Department of Electronic and Electrical Engineering, University College of London, \\ Torrington Place WC1E 7JE, London, United Kingdom; \\ ${ }^{b}$ Microelectronics Sciences Laboratories, Columbia University, NY 10027, New York, USA
}

\begin{abstract}
We demonstrate enhanced conversion efficiency $(\mathrm{CE})$ and parametric amplification of optical pulses via quasiphase-matched four-wave-mixing (FWM) in long-period Bragg waveguides made of silicon. Our study is based on a rigorous theoretical model that describes optical pulse dynamics in a periodically, adiabatically modulated silicon photonic waveguide and a comprehensive set of numerical simulations of pulse interaction in such gratings. More specifically, our theoretical model takes into account all of the relevant linear and nonlinear optical effects, including free-carriers generation, two-photon absorption, and self-phase modulation, as well as modal frequency dispersion up to the fourth-order. Due to its relevance to practical applications, a key issue investigated in our work is the dependence of the efficiency of the FWM process on the waveguide parameters and the operating wavelength. In particular, our analysis suggests that by varying the waveguide width by just a few tens of nanometers the wavelengths of the phase-matched waves can be shifted by hundreds of nanometers. Our numerical simulations show also that, in the anomalous group-velocity dispersion regime, a $\mathrm{CE}$ enhancement of more than $20 \mathrm{~dB}$, as compared to the case of a waveguide with constant width, can be easily achieved.
\end{abstract}

Keywords: Nonlinear optics, four-wave-mixing, wavelength conversion, Bragg waveguides.

\section{INTRODUCTION}

Four-wave-mixing (FWM) has attracted significant research interest, primarily due to its use in many applications in optics and photonics, including optical amplification, all-optical signal processing, and wavelength multiplexing. In a generic FWM process a pair of photons at two pump frequencies, $\omega_{p 1}$ and $\omega_{p 2}$, combine and generate two photons at signal and idler frequencies, $\omega_{s}$ and $\omega_{i}$, respectively. When the frequencies of the two pumps are equal, $\omega_{p 1}=\omega_{p 2} \equiv \omega_{p}$, this process is called degenerate FWM. In the case of optical devices with large cubic nonlinearity, such as silicon wire waveguides, FWM can become particularly strong, allowing for efficient transfer of information between the signal and idler frequencies or parametric amplification of optical signals. Driven by these appealing applications, FWM has been studied extensively in a multitude of optical guiding systems, including nonlinear optical fibers, ${ }^{1,2}$ photonic crystal fibers, ${ }^{3,4}$ and more recently silicon photonic wires. ${ }^{5,6,7,8,9,10,11}$ This latter class of nonlinear photonic devices is particularly suitable for bringing FWM based applications to chip-scale integration level, both due to the strong field confinement allowed by sub-micron silicon photonic nanowires (Si-PNWs), ${ }^{12,13}$ as well as the large third-order nonlinear susceptibility of silicon. It is chiefly for these reasons that key functionalities based on FWM in nonlinear photonic devices have already been implemented on a silicon platform, including optical parametric amplifiers, ${ }^{14,15}$ broadband frequency converters, ${ }^{16,17,18}$ sources of quantum-correlated photon pairs, ${ }^{19}$ and optical signal regenerators. ${ }^{20}$

The parameter that affects the most the efficiency of FWM is the wave vector mismatch, $\Delta \kappa$, defined as the difference between the wave vectors of the generated and annihilated photons involved in the parametric process. For waveguides with transverse size much larger than the operating wavelength, such as optical fibers, the wave vector mismatch depends mainly on the intrinsic optical constants of the medium and, consequently, it can be difficult to achieve the optimum operational regime of zero phase-mismatch. To alleviate this problem,

Corresponding author: Spyros Lavdas, E-mail: spyros.lavdas.12@ucl.ac.uk

Silicon Photonics and Photonic Integrated Circuits IV, edited by Laurent Vivien, Seppo Honkanen,

Lorenzo Pavesi, Stefano Pelli, Proc. of SPIE Vol. 9133, 91330L · @ 2014 SPIE

CCC code: $0277-786 \mathrm{X} / 14 / \$ 18 \cdot$ doi: $10.1117 / 12.2052249$

Proc. of SPIE Vol. 9133 91330L-1 
techniques based on mode matching or nonlinearly induced phase shifts have been introduced, at the cost of increased device complexity and reduced parameter space in which the device can be efficiently operated. Another possible solution is to use microstructured fibers whose modal propagation constant is strongly dependent on the device geometry, such as photonic crystal fibers. While such waveguides provide increased flexibility for tailoring the operating conditions, their potential for being used in chip-scale photonic circuits is rather limited. By contrast, due to their ultrasmall size and strong dependence of the optical modes on the geometrical and material parameters, Si-PNWs offer a convenient route towards efficient, broadly engineered phase-matched FWM processes implemented in nonlinear devices with ultrasmall footprint. In this context, recently it has been demonstrated that an additional degree of freedom can be introduced in the design of a phase-matched FWM process by simply allowing for the waveguide cross-section to vary periodically along the waveguide. ${ }^{21}$ This quasi-phase-match (QPM) technique can be used to compensate any residual wave vector mismatch and thus to achieve efficient FWM. While this QPM FWM technique has already been demonstrated in the continuous wave regime ${ }^{21}$ a pulsed operation of the device raises additional challenges. To be more specific, the FWM interaction can not be phase-matched across the entire spectrum of the co-propagating pulses. Moreover, the inherent group delay dispersion can potentially lead to additional degradation of the FWM efficiency.

In this article, we provide a comprehensive analysis of degenerate QPM FWM in pulsed regime, in the case when the pump propagates in the anomalous group-velocity dispersion (GVD) regime. The theoretical model on which our analysis of pulse dynamics is based is introduced in Sec. 2, whereas in Sec. 3 we briefly discuss the frequency dispersion and geometry dependence of the linear and nonlinear parameters of Si-PNWs considered in this work. In Sec. 4 we introduce two methods which can be used to determine the wavelengths at which the FWM process is quasi-phase-matched and compare the predictions of these two methods. Section 5 is devoted to a detailed analysis of FWM of optical pulses in uniform and Bragg waveguides, namely we investigate the dependence of the FWM gain and conversion efficiency $(\mathrm{CE})$ on the waveguide and pulse parameters. Finally, in the last section, we summarize the main conclusions of our work.

\section{THEORETICAL MODEL FOR OPTICAL PULSE PROPAGATION IN SILICON WAVEGUIDES WITH VARYING CROSS-SECTION}

Our study of pulsed QPM FWM in Si-PNWs employs a rigorous theoretical model, which takes into account the main linear and nonlinear phenomena that determine the pulse evolution. More specifically, we solve numerically a nonlinear differential equation which incorporates modal dispersive effects up to the fourth-order, self-phase modulation (SPM), two-photon absorption (TPA) and pulse self-steepening. Including all these effects allows one to describe the propagation of pulses as short as a few hundred femtoseconds. The equation describing the optical field dynamics is coupled with a standard rate equation that governs the time dependence of the freecarriers (FCs) density. This system of coupled nonlinear partial differential equation can be written as: ${ }^{9,13,22,23}$

$$
\begin{aligned}
& i \frac{\partial u}{\partial z}+\sum_{n=1}^{n=4} \frac{i^{n} \beta_{n}(z)}{n !} \frac{\partial^{n} u}{\partial t^{n}}=-\frac{i c \kappa(z)}{2 n v_{g}(z)} \alpha_{\mathrm{FC}}(z) u-\frac{\omega \kappa(z)}{n v_{g}(z)} \delta n_{\mathrm{FC}}(z) u-\gamma(z)\left[1+i \tau(z) \frac{\partial}{\partial t}\right]|u|^{2} u, \\
& \frac{\partial N}{\partial t}=-\frac{N}{t_{c}}+\frac{3 \Gamma^{\prime \prime}(z)}{4 \epsilon_{0} \hbar A^{2}(z) v_{g}^{2}(z)}|u|^{4},
\end{aligned}
$$

where $u(z, t)$ is the pulse envelope, measured in $\sqrt{W}, N(z, t)$ is the FC density, $t$ and $z$ are the time and distance along the waveguide, respectively, $\beta_{n}$ is the $n$th order dispersion parameter defined as $\beta_{n}(z)=d^{n} \beta / d \omega^{n}, \kappa(z)$ represents the overlap between the optical mode and the active area of the waveguide, $v_{g}(z)=1 / \beta_{1}(z)$ is the group-velocity, $\delta n_{\mathrm{fc}}(z)$ and $\alpha_{\mathrm{fc}}(z)$ are the FC-induced index change and FC losses, respectively, and are calculated from $\delta n_{\mathrm{fc}}(z)=-e^{2}\left(N / m_{e}^{*}+N^{0.8} / m_{h}^{*}\right) / 2 \epsilon_{0} n \omega^{2}$ and $\alpha_{\mathrm{fc}}(z)=e^{3} N(z)\left(1 / \mu_{e} m_{e}^{* 2}+1 / \mu_{h} m_{h}^{* 2}\right) / \epsilon_{0} c n \omega^{2}$, respectively, $m_{e}^{*}=0.26 m_{0}\left(m_{h}^{*}=0.39 m_{0}\right)$ is the effective mass of the electrons (holes), with $m_{0}$ the mass of the electron, and $\mu_{e}=850 \mathrm{~cm}^{2} /(\mathrm{Vs})\left[\mu_{h}=210 \mathrm{~cm}^{2} /(\mathrm{V} \mathrm{s})\right]$ is the electron (hole) mobility. The effective waveguide nonlinear coefficient, $\gamma$, is defined as $\gamma(z)=3 \omega \Gamma(z) / 4 \epsilon_{0} A(z) v_{g}^{2}(z)$ and the shock time scale is $\tau(z)=\partial \ln \gamma(z) / \partial \omega$. In our calculations we use a relaxation time, $t_{c}=1 \mathrm{~ns}$, which is much larger than the width of the pulses. Finally, $A(z)$ and $\Gamma(z)$ are the cross-sectional area and the effective third-order susceptibility of the waveguide, respectively. Here, we use the notations $z^{\prime}$ and $z^{\prime \prime}$ for the real and imaginary parts of the complex number $z$, respectively. 


\section{OPTICAL PROPERTIES OF SILICON PHOTONIC WIRE WAVEGUIDES}

The guiding device considered in this article is a silicon core with constant height, $h=250 \mathrm{~nm}$, and periodically modulated width, $w(z)$, buried in silica oxide. The $z$-dependence of the width follows a sinusiodal dependence, described by $w(z)=w_{0}+\Delta w \sin (2 \pi z / \Lambda)$, where $w_{0}, \Delta w$, and $\Lambda$ are the average waveguide width, amplitude of the width modulation, and its period, respectively [see Fig. 1(a)]. As illustrated in Fig. 1(a), we consider the case of degenerate FWM, although a second pump pulse can readily be included in our analysis. The interaction between the co-propagating pulses is chiefly affected by the waveguide dispersive properties and its nonlinear coefficients, which in turn can be determined from the mode propagation constant and the field distribution of the optical mode. The specific computational procedure used to determine the waveguide parameters and their $z$-dependence has been described in detail in our previous works. ${ }^{21,23}$
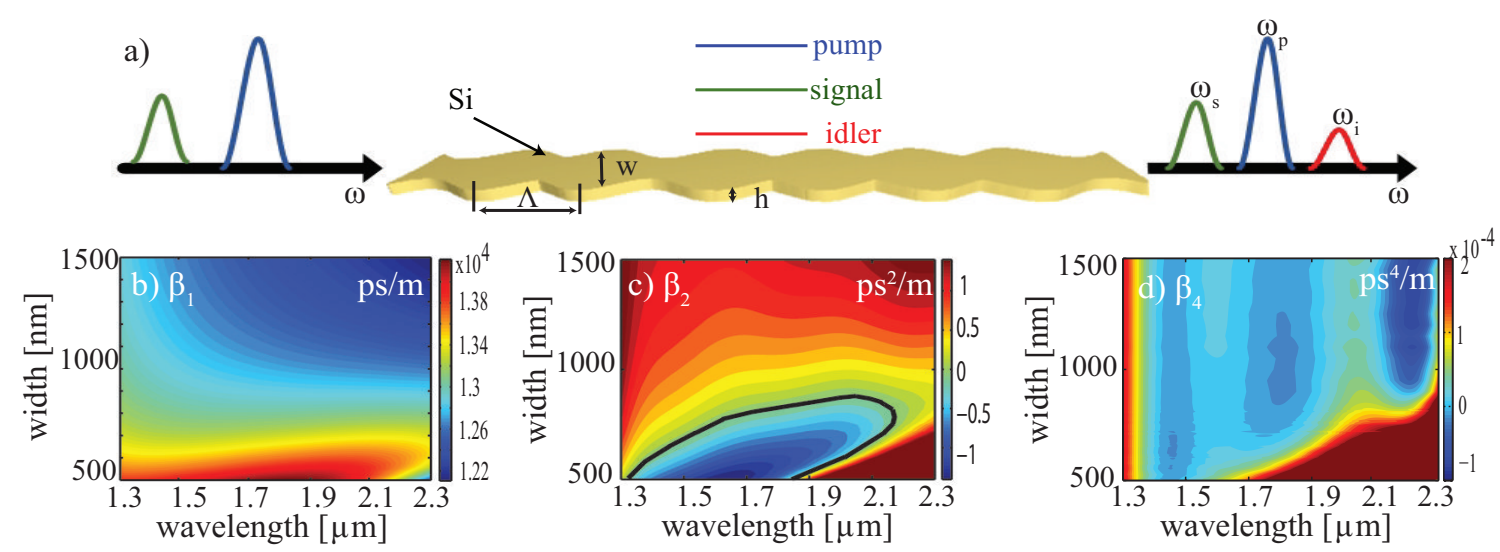

Figure 1. a) Schematics showing a periodically, width-modulated silicon nanowire. b), c), and d) show the dispersion maps of $\beta_{1}, \beta_{2}$, and $\beta_{4}$, respectively.

The dispersion properties of Si-PNWs are strongly dependent on both the operating wavelength and the width of the silicon nanowire, as it is clearly illustrated in Fig. 1, and it is this property that allows one to easily tune the working regime and efficiency of FWM in such photonic nanodevices. The dispersion map of the GVD coefficient, presented in Fig. 1(c), shows that by tuning the pump wavelength or waveguide width one can easily switch the nature of pulse propagation from the normal dispersion regime $\left(\beta_{2}>0\right)$ to anomalous dispersion $\left(\beta_{2}<0\right)$. For small width, $w \leq 887 \mathrm{~nm}$, and wavelengths, $\lambda \leq 2.187 \mu \mathrm{m}$, the GVD coefficient $\beta_{2}$ can have large (in absolute value) negative values. The normal and anomalous dispersion domains are separated by a zero-dispersion curve, depicted in Fig. 1(c) by a black line. Note that the efficiency of FWM depends only on the even terms in the Taylor series expansion of $\beta(\omega)$, and therefore close to the zero-dispersion curve the effect of the fourth-order dispersion coefficient, $\beta_{4}$, shown in Fig. 1(d), must be taken into account. Equally important in the context of pulsed FWM is the dispersion of the group velocity coefficient, $\beta_{1}$, which is presented in Fig. 1(b). Thus, for an efficient FWM one needs to maximize the duration over which the pulses overlap, which can be achieved by tuning the frequencies of the co-propagating pulses such that the corresponding group delay is small.
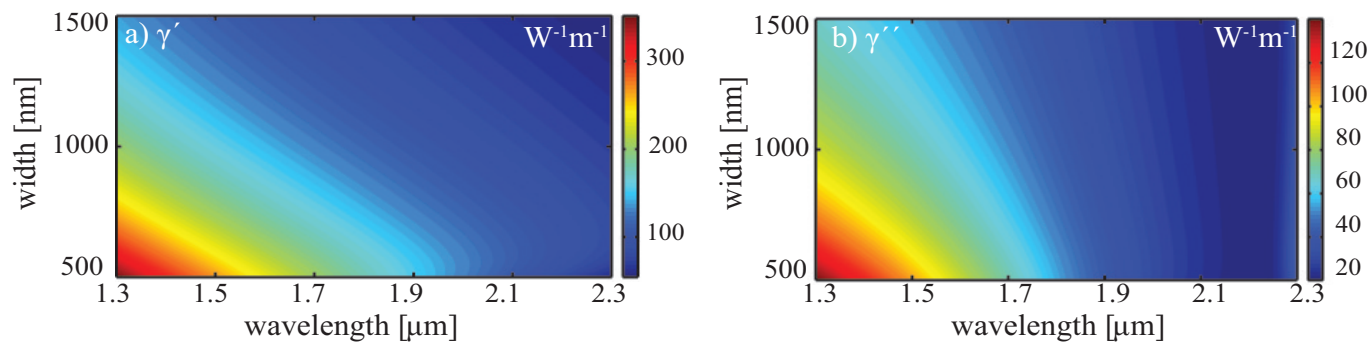

Figure 2. Dispersion maps of a) SPM coefficient, $\gamma^{\prime}$, and b) TPA coefficient, $\gamma^{\prime \prime}$. 
In the presence of the waveguide nonlinearity the mode propagation constant experiences a nonlinear shift, which is proportional to the nonlinear coefficient, $\gamma^{\prime}$, and thus the wave vector mismatch becomes dependent on the peak power of the interacting pulses. In turn, the pulse power is affected by the TPA coefficient, $\gamma^{\prime \prime}$. This suggests that a full description of the nonlinear properties of the waveguide is a prerequisite to achieving a complete characterization of FWM. To this end, the dispersion maps of both the real and imaginary parts of the waveguide nonlinear coefficient are presented in Fig. 2. Note that these coefficients are strongly dependent on the waveguide width and wavelength, increasing as the width and wavelength decrease.

\section{SPECTRAL DIAGRAMS OF DEGENERATE QPM FWM PROCESSES}

The wavelengths of the optical pulses participating in a QPM FWM process can be readily determined from the relations expressing the energy and momentum (wave vector) conservation. For the case of degenerate FWM, the energy conservation is expressed as $2 \omega_{p}=\omega_{s}+\omega_{i}$, whereas the momentum conservation relation can be written as $|\Delta \kappa| \equiv\left|2 \bar{\beta}_{p}-\bar{\beta}_{s}-\bar{\beta}_{i}\right|=K_{g}$. Here, $K_{g}=2 \pi / \Lambda$ is the grating wave vector and $\bar{\beta}_{p, s, i}$ are the nonlinear wave vectors of the interacting pulses. In a usual experimental setup the nonlinear shift of the wave vectors of the signal and idler are negligible, so that $\bar{\beta}_{s, i} \approx \beta_{s, i}$, and $\bar{\beta}_{p}=\beta_{p}-\gamma^{\prime} P_{p}$, where $P_{p}$ is the pump peak power. Under these conditions, the phase-matching condition can be written as:

$$
\left.\mid 2\left(\beta_{p}-\gamma^{\prime} P_{p}\right)-\beta_{s}-\beta_{i}\right) \mid=\frac{2 \pi}{\Lambda} .
$$

Note that in this equation all width-dependent quantities are evaluated at $w=w_{0}$. If $\Delta \omega=\omega_{s}-\omega_{p}=\omega_{p}-\omega_{i}$ is small compared to $\omega_{p}$, Eq. (2) can be brought to a form that makes it more suitable for finding the wavelengths of the quasi-phase-matched pulses by expanding in Taylor series the wave vectors $\beta_{p, s, i}(\omega)$, around the pump frequency, $\omega_{p}$. Keeping the terms up to the fourth-order, Eq. (2) becomes:

$$
\left|2 \gamma^{\prime} P_{p}+\beta_{2, p} \Delta \omega^{2}+\frac{1}{12} \beta_{4, p} \Delta \omega^{4}\right|=\frac{2 \pi}{\Lambda} .
$$

The wavelength diagrams obtained by using Eq. (2) and Eq. (3) are presented in Figs. 3(a) and 3(b), respectively. These results correspond to two Si-PNWs with $\Lambda=6 \mathrm{~mm}, P_{p}=200 \mathrm{~mW}$ and different values of the average waveguide width, $w_{0}$. The diagrams presented in Fig. 3 provide clear evidence of the strong width dependence of the two phase matching conditions. Moreover, it can be seen that, as expected, when $\Delta \omega$ is small the two methods lead to very similar predictions. On the other hand, when $\Delta \omega$ increases, the difference between the results predicted by the two phase matching conditions becomes significant, which means that more terms must be retained in the series expansions of the wave vectors of the pulses. As a consequence, in this regime of increased $\Delta \omega$, one must include additional dispersion terms in Eq. (1a) or even replace it altogether with a system of coupled equations, each of them describing one of the co-propagating pulses.
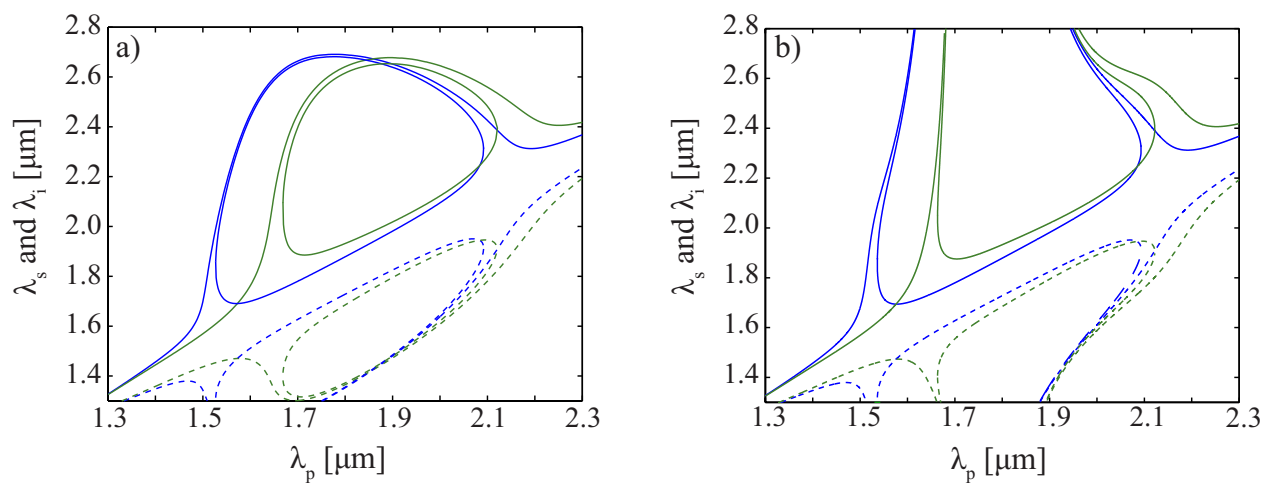

Figure 3. Phase matching diagrams showing the dependence of $\lambda_{s}$ and $\lambda_{i}$ on $\lambda_{p}$. Dashed and solid lines correspond to the signal and idler, respectively, whereas the blue and green lines correspond to $w_{0}=700 \mathrm{~nm}$ and $w_{0}=780 \mathrm{~nm}$, respectively. The diagrams in a) and b) were determined by using Eq. (2) and Eq. (3), respectively. 


\section{FWM PULSED INTERACTION IN UNIFORM AND BRAGG WAVEGUIDES}

The most convenient way to analyze the efficiency of the wavelength conversion and parametric amplification of pulses via QPM FWM in Bragg Si-PNWs is to determine the spectral content of the optical pulse, at the phase-matched frequencies. More specifically, we consider pulses whose initial temporal profile, $u(0, t)=$ $\sqrt{P_{p}}\left[\exp \left(-t^{2} / 2 T_{0}^{2}\right)+\sqrt{\varrho} \exp \left(-t^{2} / 2 T_{0}^{2}-i \Delta \omega t\right)\right]$, is the superposition of a pump pulse and a weak signal whose frequency is shifted by $\Delta \omega$. The parameter $\varrho=P_{s} / P_{p}$ is set to $10 \%$ and $1 \%$ in the cases of wavelength conversion and parametric amplification, respectively, so that in the latter case the signal is too weak to affect the pump and thus it acts as a probe. In this study we assume that the signal and the pump have the same width, $T_{0}=500 \mathrm{fs}$, although our analysis can be easily extended to the more general case of pulses with different width.

The main characteristics of QPM FWM can be revealed by comparing the propagation of a pulse in a SiPNW with uniform cross-section with the evolution of the same pulse in a Bragg Si-PNW whose period is chosen such that the phase-matching condition is satisfied. More specifically, the width of the uniform Si-PNW was $w_{0}=780 \mathrm{~nm}$ whereas in the case of the Bragg waveguide $w_{0}=780 \mathrm{~nm}, \Delta w=30 \mathrm{~nm}$, and $\Lambda=6 \mathrm{~mm}$. In both cases, $P_{p}=200 \mathrm{~mW}, P_{s}=20 \mathrm{~mW}, \lambda_{p}=1711 \mathrm{~nm}$, and $\lambda_{s}=1573 \mathrm{~nm}$, which means that if the FWM process is phase-matched an idler pulse is expected to form at $\lambda_{i}=1876 \mathrm{~nm}$. The pulse propagates in the anomalous GVD regime, both in the case of uniform and Bragg waveguides (for all widths), the linear and nonlinear waveguide coefficients being $\beta_{2, p}=-0.118 \mathrm{ps}^{2} \mathrm{~m}^{-1}, \beta_{4, p}=-1.42 \times 10^{-7} \mathrm{ps}^{4} \mathrm{~m}^{-1}$, and $\gamma^{\prime}=156.3 \mathrm{~W}^{-1} \mathrm{~m}^{-1}$.
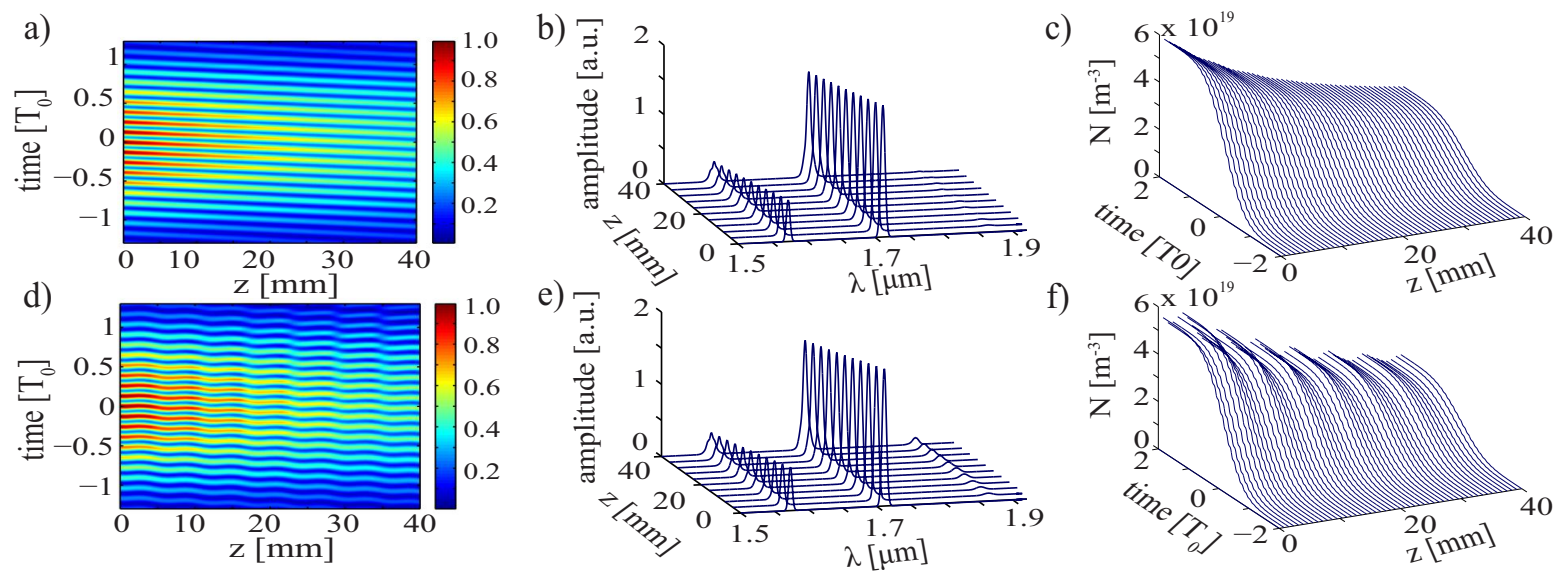

Figure 4. Top and bottom panels show the evolution of an optical pulse in a uniform and quasi-phase-matched Bragg waveguide, respectively (see the text for the values of the pulse and waveguide parameters). Left, middle, and right panels show the $z$-dependence of the temporal profile of the pulse, its spectrum, and the free-carrier density, respectively.

Figure 4 summarizes the main characteristics of the pulse propagation, namely the $z$-dependence of the temporal profile of the pulse, its spectrum, and the dynamics of the FCs. The temporal evolution of the pulse reveals two main phenomena. First, in both cases the pulse slowly moves towards its leading edge, an effect explained by the fact that the pulse group-velocity is slightly larger than the group-velocity at the reference pump frequency, $\omega_{p}$. Second, and perhaps more interestingly, the pulse propagating in the Bragg waveguide is periodically accelerated and slowed down, an effect illustrated by the undulating stripes seen in Fig. 4(d). This effect is explained by the periodic variation along the waveguide of the width, which in turn amounts to a periodic dependence on $z$ of the pulse group-velocity. More relevant for the understanding of the properties of pulsed FWM in Si-PNWs are the spectra shown in Figs. 4(b) and 4(e), which correspond to pulse propagation in uniform and Bragg waveguides, respectively. These figures clearly illustrate the differences between the pulse dynamics in the two cases, the most important one being that a much more effective energy transfer from the pump to the signal and idler is achieved in the case of QPM FWM. In particular, it can be seen that the growth rate of the idler energy is much larger in the case of the Bragg waveguide as compared to the idler build up rate in the waveguide with constant width. This important quantitative difference is better illustrated in Fig. 5, where we present the frequency profiles of the pump, signal, and idler at a propagation distance $z=40 \mathrm{~mm}$, 

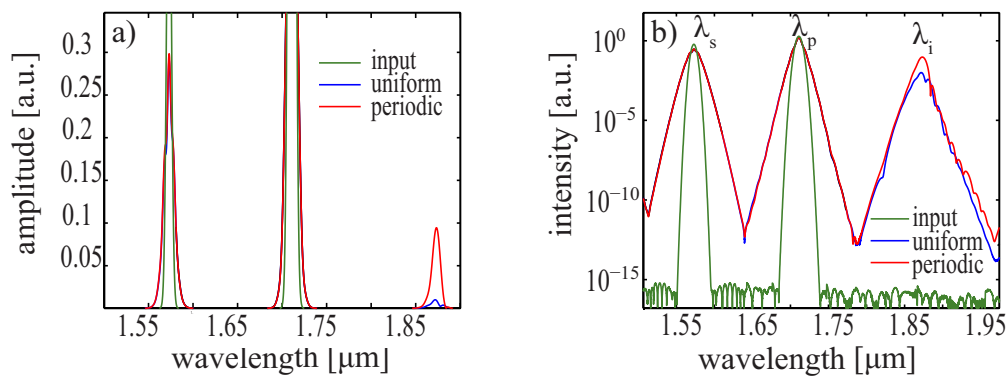

Figure 5. a) Input and output spectra (amplitude) of a pulse that propagates in a uniform and Bragg waveguide, computed at $z=40 \mathrm{~mm}$. b) Intensity of the same pulse spectra, represented in a logarithmic scale. In case of the Bragg waveguide (red curve) the idler is enhanced by more than 10 times as compared to the case of the uniform waveguide (blue curve).

for both the uniform and Bragg waveguides. Indeed, it can be seen in this figure that whereas in the case of a uniform waveguide the idler peak power is rather negligible, in the case of the Bragg waveguide it builds up to a level comparable to that of the signal. Moreover, a common characteristic of the pulse evolution is the gradual decrease of its peak power, the signature of this phenomenon being present in the FC dynamics as well. Thus, the $z$-variation of the FC density induced by a pulse propagating in the uniform and Bragg waveguides, plotted in Figs. 4(c) and 4(f), respectively, shows an overall decreasing trend. In the case of the Bragg waveguide, however, one can also observe a series of oscillations with respect to the propagation distance, $z$, which are due to the periodic variation with $z$ of the linear and nonlinear waveguide parameters. ${ }^{24}$
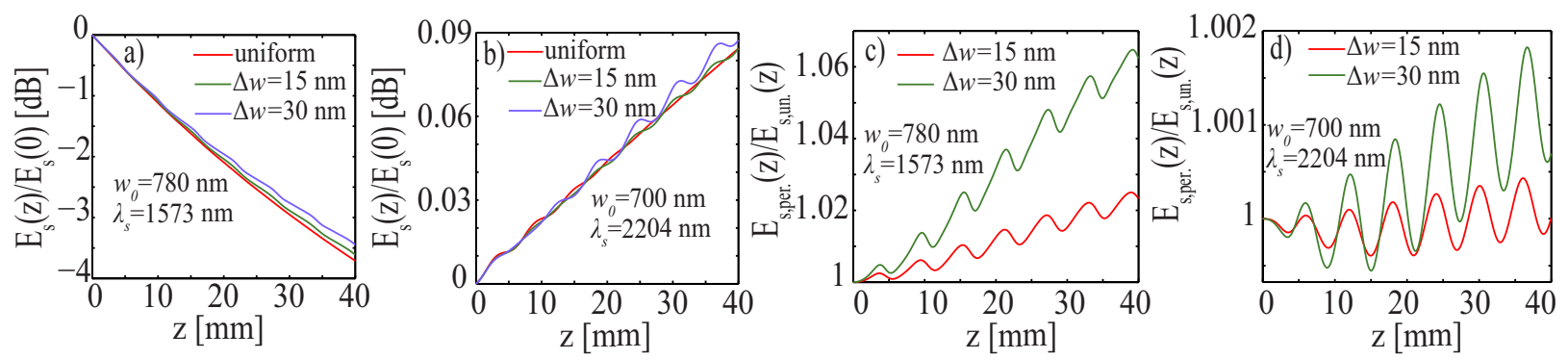

Figure 6. a), b) FWM gain vs. propagation distance, $z$, calculated in the regime of strong (telecom band) and weak (mid-IR spectral region) TPA, respectively (see the text for the waveguide and pulse parameters). c), d) Enhancement of FWM gain in Bragg waveguides as compared to uniform ones, determined in the regime of strong and weak TPA, respectively. The simulation parameters in c) and d) are the same with those in a) and b), respectively.

According to the plots in Fig. 5, the idler power is enhanced when the pulse propagates in the Bragg waveguide. However, in the FWM process, for every photon created at the idler frequency there is another photon generated at the frequency of the signal, so that one expects to observe an increased amplification of the signal as well. This FWM gain is strongly dependent on the operating wavelength because the waveguide nonlinearity, the main physical quantity that defines the strength of the FWM process, shows large frequency dispersion. In order to illustrate these ideas, we determined the QPM FWM gain in two different cases. In the first case we considered the same pair of waveguides and pulse parameters used to obtain the results presented in Fig. 4 and Fig. 5. The second propagation scenario corresponds to uniform and Bragg waveguides with $w_{0}=700 \mathrm{~nm}, \Delta w=30 \mathrm{~nm}, \Lambda=6 \mathrm{~mm}$, and co-propagating pulses with $\lambda_{p}=2070 \mathrm{~nm}, \lambda_{s}=2204 \mathrm{~nm}, \lambda_{i}=1952 \mathrm{~nm}, P_{p}=200 \mathrm{~mW}$, and $P_{s}=2 \mathrm{~mW}$. The dispersive coefficients in this case are $\beta_{2, p}=-0.415 \mathrm{ps}^{2} \mathrm{~m}^{-1}$ and $\beta_{4, p}=2.24 \times 10^{-4} \mathrm{ps}^{4} \mathrm{~m}^{-1}$, whereas the SPM coefficient is $\gamma^{\prime}=111.7 \mathrm{~W}^{-1} \mathrm{~m}^{-1}$. The main difference between the two pulse propagation regimes consists in the level of nonlinear loss experienced by the signal component of the pulse, namely $\gamma^{\prime \prime}=54.15 \mathrm{~W}^{-1} \mathrm{~m}^{-1}$ at $\lambda_{s}=1573 \mathrm{~nm}$ as compared to $\gamma^{\prime \prime}=0.418 \mathrm{~W}^{-1} \mathrm{~m}^{-1}$ at $\lambda_{s}=2204 \mathrm{~nm}$. This difference is clearly reflected by the $z$-dependence of the signal energy, as per Fig. 6 . To be more specific, in the case of large TPA the signal 

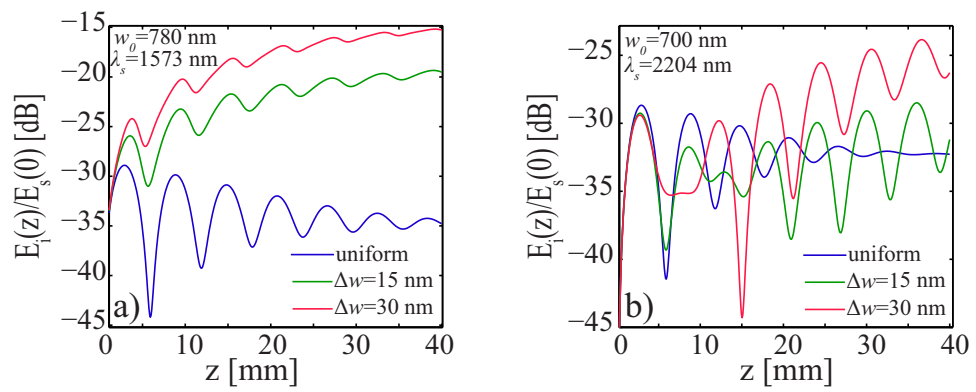

Figure 7. Conversion efficiency determined for different width-modulation amplitude, corresponding to a) $\lambda_{s}=1573 \mathrm{~nm}$ and $w_{0}=780 \mathrm{~nm}$ (strong TPA) and b) $\lambda_{s}=2204 \mathrm{~nm}$ and $w_{0}=700 \mathrm{~nm}$ (weak TPA).

energy decreases with the propagation distance [see Fig. 6(a)], whereas in the case when the nonlinear losses are weak net gain is achieved [see Fig. 6(b)]. Note, however, that in both cases the gain is larger when the pulse interaction is phase-matched and increases with the waveguide modulation amplitude, $\Delta w$ [see also Figs. $6(\mathrm{c})$ and $6(\mathrm{~d})$ ]. This dependence is explained by the fact that the nonlinear coupling between the interacting pulses, and consequently the efficiency of the FWM process, increases with $\Delta w$.

In many practical applications the main physical quantity of interest is the conversion efficiency of FWM and not the gain. Thus, the optical nonlinearity that mediates the FWM interaction leads essentially to instantaneous optical response of the medium and therefore the generated idler exactly maps the optical signal. This simple interaction mechanism allows one to convert optical signals from the signal to the idler frequencies. In order to quantify the CE of QPM FWM, we have calculated the ratio $\eta=10 \log \left[E_{i}(z) / E_{s}(0)\right]$, where $E_{i}(z)$ and $E_{s}(0)$ are the $z$-dependent idler energy and the input signal energy, respectively. The results of these calculations are presented in Figs. 7(a) and 7(b) and correspond, respectively, to the strong and weak TPA regimes discussed above. These figures suggest that, in contrast to the case of the FWM optical gain, larger CE can be achieved in the strong TPA regime. The reason for this counterintuitive result is that the peak power of the idler is relatively small and therefore it is significantly less affected by a nonlinear effect, namely the TPA. In addition, in the regime of strong TPA, the SPM coefficient, $\gamma^{\prime}$, is large as well, so that the strength of the FWM is enhanced. On the other hand, similarly to the optical gain, the CE is significantly enhanced in Bragg (phase-matched) waveguides as compared to the uniform ones, and this enhancement increases with $\Delta w$. In particular, as per Fig. 7(a), more than $20 \mathrm{~dB}$ increase in the $\mathrm{CE}$ can be achieved by employing a Bragg waveguide.

\section{CONCLUSIONS}

In conclusion, we demonstrated that efficient FWM can be achieved in Bragg silicon waveguides with period of a few millimeters. Our study showed that the conversion efficiency and FWM gain in quasi-phase-matched waveguides increases considerably as compared to the case of uniform waveguides, which proves the practical relevance of the QPM technique. Moreover, our analysis revealed that nonlinear optical losses affect the FWM gain and conversion efficiency in markedly different ways, namely whereas larger gain can be achieved in the weak TPA regime, the conversion efficiency is larger in the case of strong TPA. Finally, we investigated the dependence of the efficiency of the FWM process on the waveguide and pulse parameters, the main conclusion being that the larger the amplitude of the waveguide width modulation the more effective is the FWM interaction.

\section{ACKNOWLEDGMENTS}

The work of S. L. was supported through a UCL Impact Award graduate studentship. R. R. G. acknowledges support from the Columbia Optics and Quantum Electronics IGERT under NSF Grant DGE-1069420.

\section{REFERENCES}

[1] Stolen, R. H., Bjorkholm, J. E., and Ashkin, A., "Phase matched 3-wave mixing in silica fiber opticalwaveguides," Appl. Phys. Lett. 24, 308-310 (1974). 
[2] Agrawal, G. P., Nonlinear Fiber Optics, Academic Press, San Diego, USA (2007).

[3] Sharping, J. E., Fiorentino, M., Coker, A., Kumar, P., and Windeler, R. S., "Four-wave mixing in microstructure fiber," Opt. Lett. 26, 1048-1050 (2001).

[4] Coen, S., Chau, A. H. L., Leonhardt, R., Harvey, J. D., Knight, J. C., Wadsworth, W. J., and Russell, P. St. J., "Supercontinuum generation by stimulated Raman scattering and parametric four-wave mixing in photonic crystal fibers," J. Opt. Soc. Am. B. 19, 753-764 (2002).

[5] Claps, R., Raghunathan, V., Dimitropoulos, D., and Jalali, B., "Anti-Stokes Raman conversion in silicon waveguides," Opt. Express 11, 2862-2872 (2003).

[6] Fukuda, H., Yamada, K., Shoji, T., Takahashi, M., Tsuchizawa, T., Watanabe, T., Takahashi, J., and Itabashi, S., "Four-wave mixing in silicon wire waveguide," Opt. Express 13, 4629-4637 (2005).

[7] Espinola, R., Dadap, J., Osgood, R. M., McNab, S., and Vlasov, Y., "C-band wavelength conversion in silicon photonic wire waveguides," Opt. Express 13, 4341-4349 (2005).

[8] Panoiu, N. C., Chen, X., and Osgood, R. M., "Modulation instability in silicon photonic nanowires," Opt. Lett. 31, 3609-3611 (2006).

[9] Panoiu, N. C., Liu, X., and Osgood, R. M., "Self-steepening of ultrashort pulses in silicon photonic nanowires," Opt. Lett. 34, 947-949 (2009).

[10] Kuyken, B., Liu, X. P., Osgood, R. M., Baets., R., Roelkens, G., and Green, W. M. J., "Mid-infrared to telecom band supercontinuum generation in high nonlinear silicon-on-insulator wire waveguides " Opt. Express 19, 20172-20181 (2011).

[11] Liu, X., Kuyken, B., Roelkens G., Baets, R., Osgood, R. M., and Green, W. M. J., "Bridging the midinfrared-to-telecom gap with silicon nanophotonic spectral translation" Nat. Photonics 6, 667-671 (2012).

[12] Lin, Q., Painter, O. J., and Agrawal, G. P., "Nonlinear optical phenomena in silicon waveguides: Modelling and applications," Opt. Express 15, 16604-16644 (2007).

[13] Osgood, R. M., Panoiu, N. C., Dadap, J. I., Liu, X., Chen, X., Hsieh, I-W., Dulkeith, E., Green, W. M. J., and Vlassov, Y. A., "Engineering nonlinearities in nanoscale optical systems: physics and applications in dispesion-engineered silicon nanophotonic wires," Adv. Opt. Photon. 1, 162-235 (2009).

[14] Foster, M. A., Turner, A. C., Sharping, J. E., Schmidt, B. S., Lipson, M., and Gaeta, A. L., "Broad-band optical parametric gain on a silicon photonic chip," Nature 441, 960-963 (2006).

[15] Liu, X., Osgood, R. M., Vlasov, Y. A., and Green, W. M. J., "Mid-infrared optical parametric amplifier using silicon nanophotonic waveguides," Nat. Photonics 4, 557-560 (2010).

[16] Yamada, K., Fukuda, H., Tsuchizawa, T., Watanabe, T., Shoji, T., and Itabashi, S., "All-Optical Efficient Wavelength Conversion Using Silicon Photonic Wire Waveguide," IEEE Photon. Technol. Lett. 18, 10461048 (2006).

[17] Kuo, Y-H, Rong, H., Sih, V., Xu, S., Paniccia, M., and Cohen, O., "Demonstration of wavelength conversion at $40 \mathrm{~Gb} / \mathrm{s}$ data rate in silicon waveguides," Opt. Express 14, 11721-11726 (2006).

[18] Foster, M. A., Turner, A. C., Salem, R., Lipson, M., and Gaeta, A. L., "Broad-band continuous wave parametric wavelength conversion in silicon nanowaveguides," Opt. Express 15, 12949-12958 (2007).

[19] Lin, Q., and Agrawal, G. P., "Silicon waveguides for creating quantum-correlated photon pairs," Opt. Lett. 31, 3140-3142 (2006).

[20] Salem, R., Foster, M. A., Turner, A. C., Geraghty, D. F., Lipson, M., and Gaeta, A. L., "Signal regeneration using low-power four-wave mixing on silicon chip," Nat. Photonics 2, 35-38 (2008).

[21] Driscoll, J. B., Ophir, N., Grote, R. R., Dadap, J. I., Panoiu, N. C., Bergman, K., and Osgood, R. M., "Width Modulation of Si photonic wires for quasi-phase-matching of four wave mixing: experimental and theoretical demonstration," Opt. Express 20, 9227-9242 (2012).

[22] Chen, X., Panoiu, N. C., and Osgood, R. M., "Theory of Raman-mediated pulsed amplification in siliconwire waveguides," IEEE J. Quantum Electron. 42, 160-170 (2006).

[23] Lavdas, S., Driscoll, J. B., Jiang, H., Grote, R. R., Osgood, R. M., and Panoiu, N. C., "Generation of parabolic similaritons in tapered silicon photonic wires: comparison of pulse dynamics at telecom and mid-IR wavelengths," Opt. Lett. 38, 3953-3956 (2013).

[24] Lavdas, S., Driscoll, J. B., Grote, R. R., Osgood, R. M., and Panoiu, N. C., "Pulse compression in adiabatically tapered silicon photonic wires," Opt. Express 22, 6296-6312 (2014). 